\title{
Población y uso de hábitat del venado de páramo Odocoileus lasiotis (Artiodactyla: Cervidae) en Venezuela
}

\author{
Misael Molina y José H. Arias ${ }^{1}$ \\ 1 Universidad de Los Andes, Facultad de Ciencias Forestales y Ambientales, Escuela Técnica Superior Forestal, Mérida 5101, \\ Venezuela. Fax: 058-74401630. E-mail: mmolina@forest.ula.ve
}

Recibido 05-III-1997. Corregido 21-V-1998. Aceptado 16-VI-1998.

\begin{abstract}
Direct observations of Paramo's white-tailed deer were made along transect in the Mucubají area (Parque Nacional Sierra Nevada, Mérida, Venezuela). Sex ratio was two does per buck. Group composition varied with time of year and reproductive condition. The reproductive cycle was similar to that of the white-tailed deer from the Costa Rican dry Pacific region. Activity was maximum between $9-11 \mathrm{~h}$ and $15-17 \mathrm{~h}$; and minimum at night. The deer feed on 13 species of vascular plants (five families), and one of mushroom (Boletaceae: Agaricales). Fifteen plant species were used for shelter. Territory delimitation and signs for social communication were made primarily on gymnosperms (Cupressus and Pinus). Most deer were observed in terrain with slopes under 20\% where the more complex and diverse vegetation associations exist.
\end{abstract}

Key words: Activity periods, antler rubbing, food, paramo, Venezuela, white-tailed deer.

El venado de páramo Odocoileus lasiotis (Osgood 1914), aunque protegido mediante veda indefinida, se encuentra amenazado de extinción y sobrevive en unos pocos reductos de los Andes Venezolanos. La depredación por perros cimarrones, la cacería furtiva y la destrucción del hábitat, son sus principales amenazas (Molina 1996). La información publicada sobre este cérvido es muy escasa. Rubio (1981) presentó nociones preliminares sobre su distribución, relaciones con el hábitat, comportamiento y aspectos básicos de su biología reproductiva. Molina (1996) revisó la taxonomía de los Odocoileus de Venezuela, asignando al venado de páramo la ubicación taxonómica aquí presentada. Este autor además aportó información novedosa sobre la historia natural de este venado.
En el presente trabajo se estudia para una población del venado de páramo, (1) la proporción de sexos, (2) el tamaño y la composición de los grupos sociales, (3) algunos aspectos de la reproducción, (4) los picos de actividad, (5) el uso de la vegetación para alimento, refugio, delimitación de territorios y comunicación social, así como (6) el efecto de la inclinación del terreno sobre la actividad de los venados.

El área de estudio se ubicó en el Páramo de Mucubají ( $8^{\circ} 47^{\prime} \mathrm{N}$ y $70^{\circ} 48^{\prime} \mathrm{W}$ ), Parque Nacional Sierra Nevada, Estado Mérida, Venezuela. Mucubají tiene un período frío y seco (diciembre - mayo) y uno lluvioso (abril - octubre), siendo noviembre un mes de transición. Los promedios anuales de temperatura oscilan entre $-6^{\circ} \mathrm{C}$ y $5^{\circ} \mathrm{C}$ con una media diaria de $5.4^{\circ} \mathrm{C}$, el promedio de 
precipitaciones está alrededor de los $1000 \mathrm{~mm}$ (Azocar y Monasterio 1980). La vegetación nativa del área presenta variaciones locales: en las partes húmedas de los valles dominan las gramíneas, en las laderas predomina el rosetal de Espeletia y en los sitios rocosos y morrenas de los valles existen pequeños bosques de "Coloradito" (Polylepis sericea).

Desde diciembre de 1994 hasta mayo de 1995 se realizaron recorridos a pie que abarcaron el período comprendido entre las 7 y las 19 h., a través de cuatro transectos de $6 \mathrm{~km}$ de largo y de aproximadamente $0.2 \mathrm{~km}$ de ancho. Los transectos se ubicaron siguiendo los puntos cardinales, tomando como centro el Refugio de la Laguna Negra localizado $1 \mathrm{~km}$ al este de la Laguna de Mucubají. Una persona recorrió cada transecto a una velocidad de $1-2 \mathrm{~km} / \mathrm{h}$ por lo menos una vez cada semana. Muestras de las plantas halladas o vistas al ser mordidas por los venados se identificaron en el herbario. No se observó ningún individuo del género Mazama y no existen reportes de su presencia allí. Por otro lado, las mordidas de caballos y vacas sobre la vegetación, se pueden distinguir por su escasa selectividad y por ocasionar cortes profundos. Los venados de páramo son más selectivos y ocasionan cortes menos profundos. El esfuerzo de trabajo total fue de $711 \mathrm{hr}-$ hombre.

Para definir la composición de los grupos sociales se utilizaron datos cualitativos recopilados por los autores entre febrero de 1993 y mayo de 1995.

Se marcaron las plantas observadas con desgarramientos de corteza ocasionados por los venados al pulir sus astas y delimitar sus territorios. A 36 de estas plantas se les tomaron las medidas indicadas en el Cuadro 2. Se midió el diámetro del área descortezada en: (a) el extremo inferior, (b) el centro y, (c) el borde superior de la misma. $\mathrm{Pa}-$ ra evaluar si la magnitud de las variables medidas cambia con la especie de planta, se utilizó el análisis de varianza de una vía.

Se clasificó la inclinación del terreno en las siguientes categorías: (1) 0-20\%, (2) $21-40 \%$, (3) $41-60 \%$, y (4) $61-80 \%$.

El número total de machos y hembras juveniles observados durante los seis meses fue bajo (ocho y seis, respectivamente) si se compara con el número de adultos (30 y 83 , respectivamente) observados durante el mismo período (Cuadro 1). De la misma manera, se observaron solamente 19 crías. El bajo número de cervatillos y venados juveniles observados puede deberse a: (1) la depredación por perros cimarrones que afecta especialmente a los venados de escasa edad y (2) que tales venados son difíciles de observar debido a que se ocultan entre la vegetación durante los primeros días de vida. Si se excluye el problema de diferenciación a larga distancia entre juveniles y adultos, la población parece tener una composición de edades caracterizada por un $79.64 \%$ de adultos, $8.98 \%$ de juveniles y $11.38 \%$ de cervatillos. Durante todos los meses (excepto febrero) se observaron cervatillos de temprana edad, lo que indica que éstos pueden nacer durante todo el período. En todos los casos se observó solamente un cervatillo por cada venada lo que podría estar reflejando que normalmente nace sólo una cría por parto. La proporción de sexos fue un macho/dos hembras. Entre abril y junio fue frecuente observar machos solitarios y grupos pequeños de hembras; entre julio y septiembre se encontraron parejas de hembras y machos. Entre septiembre y octubre se observaron grupos de dos o tres hembras preñadas. El ciclo reproductivo parece seguir un patrón similar al presentado por Rodríguez y Solís (1987) para los O. virginianus del Pacífico Seco de Costa Rica.

Asumiendo que existe una relación directa entre el número de observaciones de venados y su actividad diaria, se puede mencionar la existencia de dos picos de actividad: 9-11 y 15-17 hr (Fig. 1). Estos resultados son similares a los obtenidos por Correa-Viana y Peñaloza (1996). Aparentemente, durante la noche los venados permanecieron inactivos, ocupando sitios protegidos del viento. Los picos de actividad pueden estar relacionados con condiciones ambientales favorables para los venados como temperaturas más altas, vientos moderados, y niveles bajos de insolación. En la estación seca, los altos niveles de insolación (mediodía) y las bajas temperaturas nocturnas pueden ser los factores determinantes de los momentos de menor actividad. Otras poblaciones son muy activas durante la noche (Michael 1970, 


\section{CUADRO 1}

Número de venados observados durante la época seca, de acuerdo a sexo y edad, indicando el esfuerzo.

\begin{tabular}{|c|c|c|c|c|c|c|}
\hline Mes/año & Machos J. & Machos A. & Hembras $\mathbf{J}$. & Hembras A. & Crías sexo ind. & Esfuerzo (h \\
\hline Dic. 1994 & 3 & 7 & 0 & 17 & 5 & 145 \\
\hline Ene. 1995 & 0 & 3 & 3 & 8 & 1 & 29 \\
\hline Feb. & 0 & 0 & 1 & 2 & 0 & 102 \\
\hline Mar. & 3 & 7 & 1 & 10 & 2 & 160 \\
\hline Abr. & 1 & 5 & 0 & 16 & 7 & 188 \\
\hline May. & 1 & 8 & 1 & 30 & 4 & 87 \\
\hline Total & 8 & 30 & 6 & 83 & 19 & 711 \\
\hline
\end{tabular}

J: Juveniles; A: adultos; sexo ind.: indeterminado, imposible de identificar en el campo.

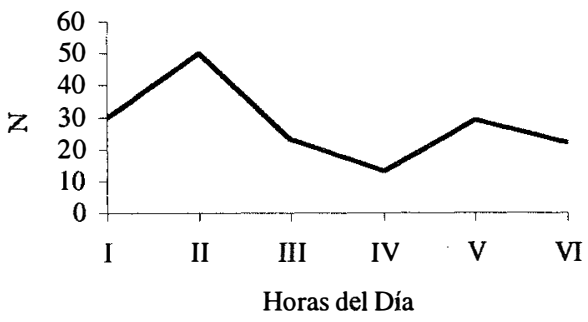

Fig. 1. Actividad diaria de los venados durante la época seca. I: 7 - 8:59; II: 9 - 10:59; III: 11:00 - 12:59; IV : 13 - 14:59; $\mathrm{V}: 15-16: 59 ; \mathrm{VI}: 17-18: 59$.

Solís 1986, Beier y McCullogh 1990, Correa-Viana 1994).

La alimentación del venado de páramo incluyó poáceas (Aciachne pulvinata, Danthonia secundiflora, Poa pauciflora y Trisetum foliosum), ciperáceas (Carex albolutescens), poligonáceas (Rumex acetosella), rosáceas (Acaena elongata, A. cylindrostrachya, Hesperomeles pernettyoides, Lachemilla hirta, y L. polylepis), oxalidáceas (Oxalis spiralis) y hongos (Boletus sp., Boletaceae:Agaricales).

El refugio estuvo representado por plantas introducidas: cupresáceas (Cupressus lusitanica) y pináceas (Pinus patula, $P$. radiata, $P$. lutea), y plantas nativas: compuestas (Bacharis prunifolia, Espeletia timotensis, E. schultzii), rosáceas (Hesperomeles pernettyoides, Polylepis sericea) y rubiáceas (Arcytophyllum caracasanum, Hypericum larcifolium, y Sisyrinchium iridifolium).

Se pudo observar marcas ocasionadas por astas de venados sobre $C$. lusutanica, $P$. patula, $P$. radiata, $P$. lutea, E. timotensis, $P$. sericea, $A$. caracasanum, y $H$. laricifolium. AIZD y AP no fueron significativamente diferentes $(0.1>p>$ $0.05, \mathrm{~F}=0.38$ ) entre las especies, pero si se encontraron diferencias al comparar el DP $(\mathrm{p}<$ $0.025, \mathrm{~F}=4.26)$ y LZD $(\mathrm{p}<0.005, \mathrm{~F}=20.27)$ entre las mismas. Los venados usan principalmente plantas de baja altura $(\overline{\mathrm{x}}=171.08 \mathrm{~cm}, \mathrm{~N}$ =36) $\mathrm{y}$ tallos delgados $(\overline{\mathrm{x}}=6.28 \mathrm{~cm}, \mathrm{~N}=36) \mathrm{pa}$ ra pulir sus astas (Cuadro 2).

\section{CUADRO 2}

Promedio $(\mathrm{cm})$ de las variables medidas en cada tipo de planta.

$\begin{array}{cccc}\text { Variable } & \text { Pinos }(\mathrm{N}=21) & \text { Hypericum } \mathrm{sp} .(\mathrm{N}=7) & \text { Arcytophylum } \mathrm{sp} .(\mathrm{N}=8) \\ \text { AIZD } & & & \\ \text { DP } & 45.33 & 32.50 & 44.43 \\ \text { LZD } & 8.24 & 3.63 & 3.43 \\ \text { AP } & 40.71 & 52.00 & 20.00 \\ & 163.76 & 208.75 & 150.00\end{array}$

AIZD: altura al inicio de la zona descortezada; DP: diámetro promedio de la zona descortezada; LZD: longitud de la zona descortezada, y AP: altura estimada de la planta 
El $77 \%$ de las observaciones corresponden a áreas con pendientes menores de $20 \%$ en las que se concentraron los venados durante los meses más secos $(20 \%$ en pendientes de 21 a $40 \%$; $2 \%$ en pendientes de 41 a $60 \%$, y $1 \%$ en pendientes de 61 a 80\%. Según Day (1964), y Suring (1979), el mayor uso de las áreas de menor pendiente se explica por la existencia de asociaciones vegetales más diversas y complejas.

Este trabajo se realizó con apoyo económico de EcoNatura. Jairo Ramírez, Alirio Nieto y José Peñaloza, guardaparques del Parque Nacional Sierra Nevada, Sector Mucubají, ayudaron en la logística durante el trabajo de campo.

\section{REFERENCIAS}

Azocar, A. \& M. Monasterio. 1980. Estudio de la variabilidad meso y microclimática en el páramo de Mucubají, p. 225-262. In M. Monasterio (ed.). Estudios ecológicos en los páramos andinos, Universidad de Los Andes, Mérida, Venezuela.

Beier, P. \& D. R. McCullogh. 1990. Factors influencing white-tailed deer activity patterns and habitat use. Wildl. Monogr. 121. The Wildlife Society, Washington, D. C. $5 \mathrm{lp}$.

Correa-Viana, M. 1994. Actividad diaria y selección de hábitat por el venado caramerudo, Odocoileus virginianus, en Masaguaral, Edo. Guárico, Venezuela. Biollania 10:33-42.

Correa-Viana, M. \& J. Peñaloza. 1996. Situación actual y ecología del venado de páramo en Mucubají (Estado Mérida, Venezuela). Biollania 11:149-152.
Danields, H. 1987. Ecología nutricionạl del venado caramerudo (Odocoileus virginianus) en los llanos centrales. Tesis Doctoral, Univ. Central de Venezuela, Caracas.

Day, G. I. 1964. An investigation of white-tailed deer (Odocoileus virginianus) forage relationships in the Chiricagua Mountains. Tesis de Maestría, Univ. de Arizona, Tuczon.

Michael, E. D. 1970. Activity patterns of white-tailed deer in south Texas. Tex. J. Sci. 21:417-428.

Molina, M. 1996. Revisión taxonómica de los Odocoileus (Mammalia, Artiodactyla, Cervidae) de Venezuela, con aportes a la historia natural y conservación del venado de páramo. Tesis de Licenciatura en Biología, Univ. de Los Andes, Mérida, Venezuela. 116 p.

Rodríguez, M. \& V. Solís. 1987. Ciclo reproductivo del venado cola blanca de la Isla de San Lucas, Costa Rica, p. 31-32. In V. Solís, M. Rodríguez y C. Vaughan (eds.). Actas del primer taller nacional sobre el venado cola blanca (Odocoileus virginianus) del Pacífico Seco, Costa Rica. 129 p.

Rubio, I. 1981. Contribución al estudio del venado de páramo (Odocoileus sp.) (Páramo de Mucubají), Mérida, Venezuela. Rev. Ecol. Conserv. Omit. Lat.1:4-7.

Solís, V. 1986. Aspectos de la ecología de las crías de venado en la Isla San Lucas, Costa Rica, p 27-29. In V. Solís, M. Rodríguez y C. Vaughan (eds.). Actas del primer taller nacional sobre el venado cola blanca (Odocoileus virginianus) del Pacífico Seco, Costa Rica.

Suring, L. H. \& P. A. Vohs Jr. 1979. Habitat use by columbian white-tailed deer. J. Wildl. Mgmt. 43:610-619. 